\title{
Guideline of guidelines: Muscle-invasive bladder cancer
}

\author{
Nicolas Pavlos Omorphos ${ }^{1}$ (1), John Carlo Pansaon Piedad ${ }^{1}$ (D), Nikhil Vas $\operatorname{dev}^{1,2}$ (1)
}

Cite this article as: Omorphos NP, Piedad JCP, Vasdev N. Guideline of guidelines: Muscle-invasive bladder cancer. Turk J Urol 21 September 2020. 10.5152/tud.2020.20337. [Epub Ahead of Print]

ORCID IDs of the authors: N.P.O. 0000-0002-2389-7450; J.C.P.P. $0000-0003-0043-352 X$ N.V. 0000-0002-3966-5734.

${ }^{1}$ Hertfordshire and Bedfordshire Urological Cancer Centre, Department of Urology, Lister Hospital, Stevenage, UK

${ }^{2} S c h o o l$ of Medicine and Life Sciences, University of Hertfordshire, Hatfield, UK

Submitted:

23.07.2020

Accepted:

10.08.2020

Available Online Date: 21.09.2020

Corresponding Author: Nikhil Vasdev

E-mail:

nikhilvasdev@doctors.org.uk

(C) Copyright 2020 by Turkish

Association of Urology

Available online at

www.turkishjournalofurology.com

\begin{abstract}
Muscle-invasive bladder cancer accounts for $25 \%$ of bladder cancer cases and represents a spectrum of disease, which can result in significant morbidity and mortality for anyone affected. Current management has evolved through years of research and clinical practice. It is based on a risk-benefit approach, which is often tailored to the individual requirements of patients and involves cystectomy, neoadjuvant and adjuvant therapies, and multimodal surveillance paradigms to achieve high survival rates. Multiple guidelines exist to assist the clinicians in this decision-making process, but their adherence is often variable. In this article, we aimed to review the 4 most commonly used guidelines from the European Association of Urology, the National Institute for Health and Care Excellence, the National Comprehensive Cancer Network, and the American Urological Association.
\end{abstract}

Keywords: Bladder cancer; guidelines; investigations; management; muscle-invasive bladder cancer; treatment.

\section{Introduction}

Bladder cancer is the $6^{\text {th }}$ most common cancer in men and the $17^{\text {th }}$ most common cancer in women. ${ }^{[1]}$ Each year, 275,000 people are diagnosed with this disease, and 108,000 die from it. In industrialized countries, $90 \%$ of bladder cancers are urothelial or transitional cell carcinomas, whereas squamous cell carcinomas are more prevalent in developing nations. The most common preventable risk factor associated with bladder cancer is smoking, and nonpreventable risk factors include increasing age and family history. Nonmetastatic bladder cancer is separated into non-muscle-invasive (75\% of cases) and muscle-invasive disease (25\% of cases).

In 2018, the worldwide age-standardized incidence rate (per 100,000) was 9.6 for men and 2.4 for women, with a cumulative risk of 1.08 for men and 0.27 for women. Overall, the highest age-standardized incidence for men was reported in Greece (40.4) and in Lebanon (9.4) for women. The age-standardized worldwide mortality was 3.2 in men and 2.3 in women. ${ }^{[2]}$ In this review, we aimed to summarize the commonly used guidelines developed for muscle-invasive bladder cancer (MIBC) from 4 prominent organizations. It is worthwhile to note that although these clinical guidelines present the best available evidence, they will not always lead to the best outcome. Clinical expertise and patient values and preferences will always have to be taken into consideration.

\section{Methods}

A guideline-focused PubMed search on the topic of MIBC was performed from 2015 to present, and the websites of urological and oncological societies were manually searched to identify the most relevant guidelines. A thorough review of the guidelines that are most commonly used in practice was also undertaken, namely the American Urological Association (AUA), the National Comprehensive Cancer Network (NCCN), the National Institute for Health and Care Excellence (NICE) and the European Association of Urology (EAU) guidelines. Each guideline was recently updated and was based on extensive literature review by an expert panel.

\section{The AUA Guideline}

The AUA guideline was last updated in 2017 , and no amendment has been published since then. [1] The panel consisted of 14 members, and the draft guideline document was distributed to 128 
peer reviewers, 67 of whom submitted comments. Where sufficient evidence existed, a strength rating of "A" (high), "B" (moderate), or "C" (low) was assigned for support of "strong," "moderate," or "conditional" recommendations. In the absence of sufficient evidence, "clinical principles" and "expert opinions" were issued.

\section{The NICE Guideline}

The NICE guideline was created by a panel of 15 members, which included medical professionals, technical experts, and lay public representatives. ${ }^{[2]}$ Non-muscle- and muscle-invasive diseases were included in the same document. The guidelines were published in February 2015 and reviewed in 2019. No subsequent evidence was deemed strong enough to amend any of the existing recommendations. The recommendations varied from "must" (signifying a legal duty), "should" (strong recommendation), and "to consider" (suggesting a case-by-case analysis).

\section{The EUA Guideline}

The EUA guideline was formed by a panel of 14 medical professionals.$^{[3]}$ The first MIBC-specific guideline was issued in 2004 and has been regularly updated since then. The latest update was in 2020. The recommendations were based on a strength rating, the basis of which was the modified Grading of Recommendations, Assessment, Development, and Evaluation methodology. This addressed a number of key factors, namely, the quality of evidence, the extent of the effect, and the balance between desirable and undesirable outcomes, among others. The strength of each recommendation was subsequently represented by the words "strong" or "weak."

\section{The NCCN Guideline}

The NCCN guideline was formed by a panel of 35 medical professionals, technical experts, and lay representatives commissioned by this USA-based Network. ${ }^{[4]}$ It was first published in 1998 and has been regularly updated subsequently, most recently, in May 2020. The guideline refers to both non-muscleand muscle-invasive diseases and uses treatment algorithms and

\section{Main Points:}

- A number of guidelines have been published on muscle-invasive bladder cancer, each suggesting a variable management plan.

- Cystoscopy is always clinically indicated in the diagnostic phase.

- Appropriate staging of the cancer is required to ensure appropriate treatment.

- Radical cystectomy often forms a part of the management plan in localized disease.

- In case of guideline discrepancy, a holistic approach catering to the individual patient characteristics is required to ensure a personalized management strategy. flowcharts to display the recommended management strategies. Evidence is categorized as "1" (high-level evidence with uniform consensus), "2A" (lower-level evidence with uniform consensus), "2B" (lower-level evidence with consensus), and " 3 " (any level of evidence with major disagreement). Categories of preference are also mentioned, ranging from "preferred intervention" to "other recommended intervention" and "useful in certain circumstances."

\section{Guideline Recommendations: Assessment and Diagnosis}

\section{Lifestyle modification}

All the guidelines acknowledge the correlation between tobacco smoking and bladder cancer and suggest that smoking cessation should be encouraged in all patients. ${ }^{[3]}$ The AUA and EAU guidelines also recognize the increased risk of bladder cancer conferred by previous radiotherapy treatments, with the EAU advising closer monitoring for younger patients. ${ }^{[4,5]}$ The EAU also recommends informing workers regarding the occupational hazards with potentially carcinogenic substances, such as aromatic amines. They also caution the clinicians to avoid pioglitazone administration in patients with previous or active bladder cancer because of increased risk of recurrence or progression. ${ }^{[5]}$

\section{Pathology}

Muscle-invasive cancers are often high-grade urothelial cancers; therefore, prognostic information is primarily obtained through identification of the morphological subtype instead of its grade. ${ }^{[6,7]}$ All the guidelines agree that the World Health Organization (WHO) grading system is to be followed but disagree on the version to be used. The NCCN follows the 2016 guideline, the AUA and EAU follow the 2004 guideline, and the NICE follows the 1973 classification. An important distinction between these WHO classifications is that the 1973 classification introduced significant ambiguity over the G2 tumor group and the grading of G1/2 and G2/3 groups, whereas the 2004 classification introduced the concept of low- and high-grade tumors in addition to a new category, the papillary urothelial neoplasm of low malignant potential. ${ }^{[5]}$ In terms of molecular markers, they do not form part of standard clinical practice despite some evidence of improved histological grading accuracy, but they may be included in future classifications..$^{[2,7]}$

There is a general consensus that pathology reports should include certain pathologic features, such as the presence of muscularis propria (detrusor muscle) and whether it has been invaded, the depth of invasion, and the presence of carcinoma in-situ (CIS) or variant histology.

\section{Diagnostic Evaluation}

The most common presenting complaint observed in patients with bladder cancer is hematuria. Other symptoms include fre- 
quency, urgency, dysuria, and, in cases of advanced tumors, pelvic pain and features of urinary tract obstruction. Once referred, the general consensus is that rectal and vaginal bimanual examination should be performed after an examination under anesthesia before or after a transurethral resection of the bladder tumor (TURBT) to assess whether there is a palpable tumor or it is fixed to the pelvic wall. ${ }^{[8]}$ The results of the clinical examination should always be interpreted with care.

\section{Cystoscopy}

All the guidelines agree that a cystoscopic evaluation of the entire urethra and bladder should be performed before any resection. Evaluation should include documentation of the tumor site, size, appearance (papillary or solid), and a description of any mucosal abnormalities ${ }^{[9]}$. However, if the tumor has already been visualized via an imaging study, the EAU guideline allows for omission of the cystoscopy, with the patient proceeding directly to TURBT for histological diagnosis. ${ }^{[5]}$

Currently, white light cystoscopy (WLC) is the gold standard recommended by the 4 major guidelines for the assessment and staging of bladder cancer. Use of photodynamic diagnosis or narrow-band imaging, in conjunction with WLC, is recommended by the NICE because of the increased sensitivity it confers for non-muscle-invasive bladder cancer (NMIBC), particularly CIS because it is more sensitive for papillary lesions and CIS. ${ }^{[10,11]}$ These novel techniques need to be performed by experienced surgeons to reduce the false-positive results. ${ }^{[1]}$

\section{Imaging}

There is a growing evidence that a combined imaging and endoscopic approach confers improved diagnostic accuracy for upper urinary tract tumors. ${ }^{[12,13]}$ EAU guidelines support this approach by advising that if a tumor has been unequivocally visualized with imaging studies, such as computed tomography (CT), magnetic resonance imaging (MRI), or ultrasound (US), the patient can proceed directly to a preoperative cystoscopy and TURBT. ${ }^{[3]}$

\section{Cytology}

Evaluation of cytology samples can be confounded by urinary tract infections, stones, and low cellular yields. However, at the hands of experienced clinicians, specificity can exceed $90 \% .^{[14]}$ A negative cytology can, therefore, never exclude a tumor, but it has high sensitivity for high-grade tumors and CIS. Examination includes analysis of the voided urine or of bladder washings for exfoliated cancer cells. Currently, there are no known markers specific for the diagnosis of invasive bladder cancer. ${ }^{[15]}$

A standardized reporting system known as the "Paris System," published in 2016, is used to characterize the diagnostic categories of urinary cytology. ${ }^{[16]}$ Per the NICE guidelines, use of any urinary biomarkers is not a substitute for cystoscopy in the preliminary stages of bladder cancer investigation or during the follow-up period after treatment unless this is in the context of a clinical research study. ${ }^{[2]}$

\section{Staging}

The treatment strategies and prognosis of bladder cancer are dependent on the tumor stage and grade. ${ }^{[21]}$ In clinical practice, the tumor, node, metastasis classification $\left(2017,8^{\text {th }}\right.$ edition) is recommended. There is also evidence that vascular and lymphatic invasion confers independent prognostic significance. ${ }^{[17,18]}$ NMIBC (formerly superficial bladder cancer) encompasses $\mathrm{Ta}, \mathrm{T} 1$, and CIS tumors, whereas T2-4 tumors are classified as MIBCs (Table 1). Histologically, a low or high grade is also attributed to urothelial carcinomas.

CT and MRI of the abdomen and pelvis are the most commonly used techniques in bladder cancer staging. They are used before TURBT to establish the extent of tumor invasion locally to the lymph nodes, upper urinary tract, or distant organs. ${ }^{[19-22]}$ There is evidence that MRI is better than CT for staging; however, neither can accurately diagnose the microscopic invasion of perivesical fat; therefore, their principal aim should be to detect T3b disease or higher. ${ }^{[22-24]}$

Where CT or MRI is used, all the guidelines agree that they should be accompanied by intravenous contrast with delayed imaging to allow for evaluation of the renal pelvis and ureters for upper urinary tract carcinomas. Evidence suggests that CT urography offers the highest diagnostic accuracy with good overall sensitivity (0.67-1.0) and specificity (0.93-0.99) for the upper urinary tract carcinomas. ${ }^{[19,25,26]}$ Additionally, if hydronephrosis is present on imaging, it is associated with advanced disease and poor oncological outcome. ${ }^{[25]}$ If CT urography is contraindicated because of renal dysfunction or iodine-based contrast allergy, MRI urography can be offered as an alternative. The caveat is that the glomerular filtration rate must be greater than $30 \mathrm{~mL}^{-1}$ and that there is no acute renal failure to avoid nephrogenic systemic fibrosis. ${ }^{[27]}$ If both CT and MRI are contraindicated, renal US or noncontrast CT can be used in conjunction with retrograde ureteropyelography per the NCCN guidelines. ${ }^{[4]}$

Furthermore, chest imaging should always be performed owing to the strong association between bladder cancer and smoking. As a minimum, a chest X-ray should be performed in nonsmokers, but ideally, a CT should be conducted in all patients owing to the increased sensitivity in evaluating metastatic cancer. ${ }^{[28]}$ This would follow the recommendations of the AUA, EAU, and NCCN.

Per the NCCN guidelines, a complete chemistry profile and complete blood count should be performed during the staging process. If alkaline phosphatase is negative and there are no symptoms or signs suggestive of bone or brain metastases, no 
further investigations are required because of the low likelihood of such metastases at the time of presentation. ${ }^{[29,30]}$ If bone me-

\section{Table 1. TNM classification of bladder cancer ${ }^{[17]}$}

\section{T - Primary Tumor}

Non-muscleinvasive disease

Tx

Tumor cannot be assessed

T0

No evidence of tumor

$\mathrm{Ta}$

Noninvasive papillary carcinoma

Tis

Carcinoma in-situ: "flat tumor"

$\mathrm{T} 1$

Subepithelial connective tissue invasion

Muscle-invasive disease

Muscularis propria muscle invasion

pT2a

Invasion of superficial muscularis propria muscle (inner half)

pT2b Invasion of deep muscularis propria muscle (outer half)

T3 Perivesical tissue invasion

T3a Microscopic invasion

T3b Macroscopic invasion (extravesical mass)

T4 Invasion of any of the following structures: prostate stroma, seminal vesicles, uterus, vagina, pelvic or abdominal wall

pT4a Prostate stroma, seminal vesicles, uterus or vagina involvement

pT4b Pelvic or abdominal wall involvement

\section{$\mathrm{N}$ : Regional lymph nodes}

$\mathrm{Nx}$

Regional lymph nodes cannot be assessed

N0 No regional lymph node metastasis

N1 Metastasis in a single lymph node in the true pelvis (hypogastric, obturator, external iliac, or presacral)

N2 Metastasis in multiple regional lymph nodes in the true pelvis (hypogastric, obturator, external iliac, or presacral)

N3 Metastasis in a common iliac lymph node(s)

M: Distant metastasis

M0

No distant metastasis

M1a Nonregional lymph nodes

M1b Other distant metastasis

TNM: tumor, node, metastasis tastases are suspected, further imaging is required. Currently, it is unclear which diagnostic modality is ideal for this, with the EAU suggesting an MRI, the AUA suggesting a bone scan, and the NCCN suggesting multiple modalities, including an MRI, a bone scan, or ${ }^{18} \mathrm{~F}$-fluorodeoxyglucose positron emission tomography (PET)/CT. ${ }^{[29,31-33]}$ Guidelines are less specific when it comes to brain metastases, but the NCCN recommends MRI with or without contrast in symptomatic or selected "high-risk" patients (e.g., small cell histology) or CT with contrast, if MRI is contraindicated. ${ }^{[30,34]}$

The role of routine PET imaging is currently undefined in the staging of bladder cancer, although some studies have demonstrated increased sensitivity in detecting pelvic lymphadenopathy nodes in advanced disease. ${ }^{[35]}$ The current consensus by all 4 guidelines is to avoid routine PET scanning during the initial staging evaluation, but to consider it; further evaluation is required in patients with abnormal findings or in whom lymph node biopsy is not feasible. ${ }^{[36]}$

\section{Transurethral Resection of Bladder Tumor}

All 4 major guidelines agree that TURBT is the most accurate and reliable technique to acquire a histopathological diagnosis and staging, if performed correctly ${ }^{[1-4]}$ All visible lesions should be resected piecemeal to ensure that a sample of the detrusor muscle is included in all the resections for appropriate staging. Each piece should ideally be labeled and packed in separate containers for analysis. The NICE further advises that random biopsies should not be taken from normal looking urothelium during TURBT, unless a specific clinical indication exists. In addition, both the NICE and the NCCN recommend a single dose of intravesical chemotherapy (gemcitabine or mitomycin C) during the TURBT (or within the next 24 hours). ${ }^{[2,4]}$ The EAU recommends that prostatic urethral biopsies should also be taken in cases of bladder neck tumors, presence or suspicion of CIS, visible prostatic urethral abnormalities, or where a positive cytology is noted in the absence of evidence of bladder tumor. If a urethral tumor is present, urethrectomy would be required, which would be a contraindication to neobladder formation.

\section{Guideline Recommendations: Treatment}

Once the bladder tumor has been appropriately staged, a multidisciplinary approach involving the patient is encouraged to formulate an appropriate management plan. Treatment options are dependent on performance status and comorbidity and include partial or radical cystectomy, neoadjuvant or adjuvant therapy, bladder-preserving approaches, systemic therapy, and, in some selected cases, TURBT only. ${ }^{[37]}$ A common comorbidity index used in bladder cancer is the age-adjusted Charlson comorbidity index, which has also been found to be an independent prognostic factor for perioperative and overall mortality and cancerspecific mortality. ${ }^{[38]}$ 


\section{Neoadjuvant therapies}

The standard curative approach for MIBC (T2-4a, cNOM0) is radical cystectomy; however, this confers a 5-year survival of only $50 \%$. As such, since the 1980 s, cisplatin-based neoadjuvant chemotherapy has been routinely used to improve outcomes (additional $8 \%$ at 5 years), unless contraindicated. ${ }^{[39]}$

Evidence on the use of neoadjuvant and/or adjuvant radiotherapy is more limited, with no clear evidence of improved survival, or that it can be used with a curative intent. There are signs, however, that use of chemoradiotherapy (radiotherapy combined with a radiosensitizer) can result in down-staging; therefore, the AUA, NICE, and NCCN recommend it in patients who are unable to undergo cystectomy. ${ }^{[1,2,4]}$ urinary tract (if multifocal disease, NMIBC with CIS, trimodal treatment, or positive ureteral margins)

Cystoscopy and/or cytology in selected patients, e.g. multifocal tumor, CIS and tumor in prostatic urethra

If trimodal treatment used, regular follow-up is required to avoid relapse

Cystoscopy

$\mathrm{CT}$ of thorax and abdomen

3-4 monthly follow-up for

3 years and then 6-monthly

6-monthly

3-4 monthly follow-up for 3 years and then 6-monthly (stopped after 5 years)

$\mathrm{pH}$ measurement (+sodium Unclear bicarbonate substitution)

Vitamin B12 levels (if bowel diversion used)

NICE CT thorax, abdomen, and pelvis

Routine bloods:

Renal function

$\mathrm{pH}$ level

Vitamin B12

Folate

\section{If defunctioned urethra in Annually for 5 years}

men:

Urethral washings for cytology and/or urethroscopy

If radical radiotherapy, routine cystoscopy follow-up is required

Rigid cystoscopy 3 months after radiotherapy is completed

Then rigid or flexible cystoscopy:

3 -monthly for 2 years 6 -monthly for a further 2 years

Yearly thereafter (dependent on clinical judgment and patient preference)

If radical radiotherapy, regular upper tract imaging is required

Yearly for 5 years

AUA: American Urological Association; EAU: European Association of Urology; NICE: National Institute for Health and Clinical Excellence; CT: computed tomography; MRI: magnetic resonance imaging; NMIBC: non-muscle-invasive bladder cancer; CIS: carcinoma in-situ.
Immunotherapy is a future alternative to chemoradiotherapy, and currently, there are ongoing clinical trials assessing its effectiveness, the results of which are promising. As such, this treatment can only be offered to patients within a trial setting at this point.

\section{Radical cystectomy}

This is the standard of treatment for localized MIBC (T2-T4a, cN0M0) in most of the developed countries and should be dure should ideally happen in high-volume centers because this is likely to improve the quality of care and reduce the perioperative morbidity and mortality. The procedure involves a cystoprostatectomy in men and a cystectomy (with or without a hysterectomy) in women after the formation of a urinary diversion (i.e., a urinary stoma or continent urinary diversion) depending on contraindications. If a biopsy of the prostatic urethra has not been taken by this time, the EAU advises that a frozen section should be obtained at this point. Bilateral regional pelvic lymph node dissection should also be performed, but there remains a lack of evidence whether a limited or extended approach should be followed ${ }^{[4]}$ Laparoinvestigation. Therefore, current best practice is open radical cystectomy.

Where possible, sexual function preserving procedures should be discussed with patients who are motivated to avoid sexual dysfunction and meet the requirements of an organ-confined disease lacking any bladder neck, urethra, or prostate involvement. ${ }^{[1]}$ In men, options include prostate, capsule, seminal, and nerve-sparing techniques; however, none have been shown to be superior so far. In women, there are less data regarding pelvic organ preservation. However, EAU guidelines suggest that it should be considered if sexual function preservation is desired. performed within 3 months of initial resection..$^{[40]}$ The procescopic and robotic-assisted laparoscopic cystectomy are alternatives to the open approach, but currently, they are still under 


\section{Table 3. Summary of the surveillance plan recommended by the $\mathrm{NCCN}^{[4]}$}

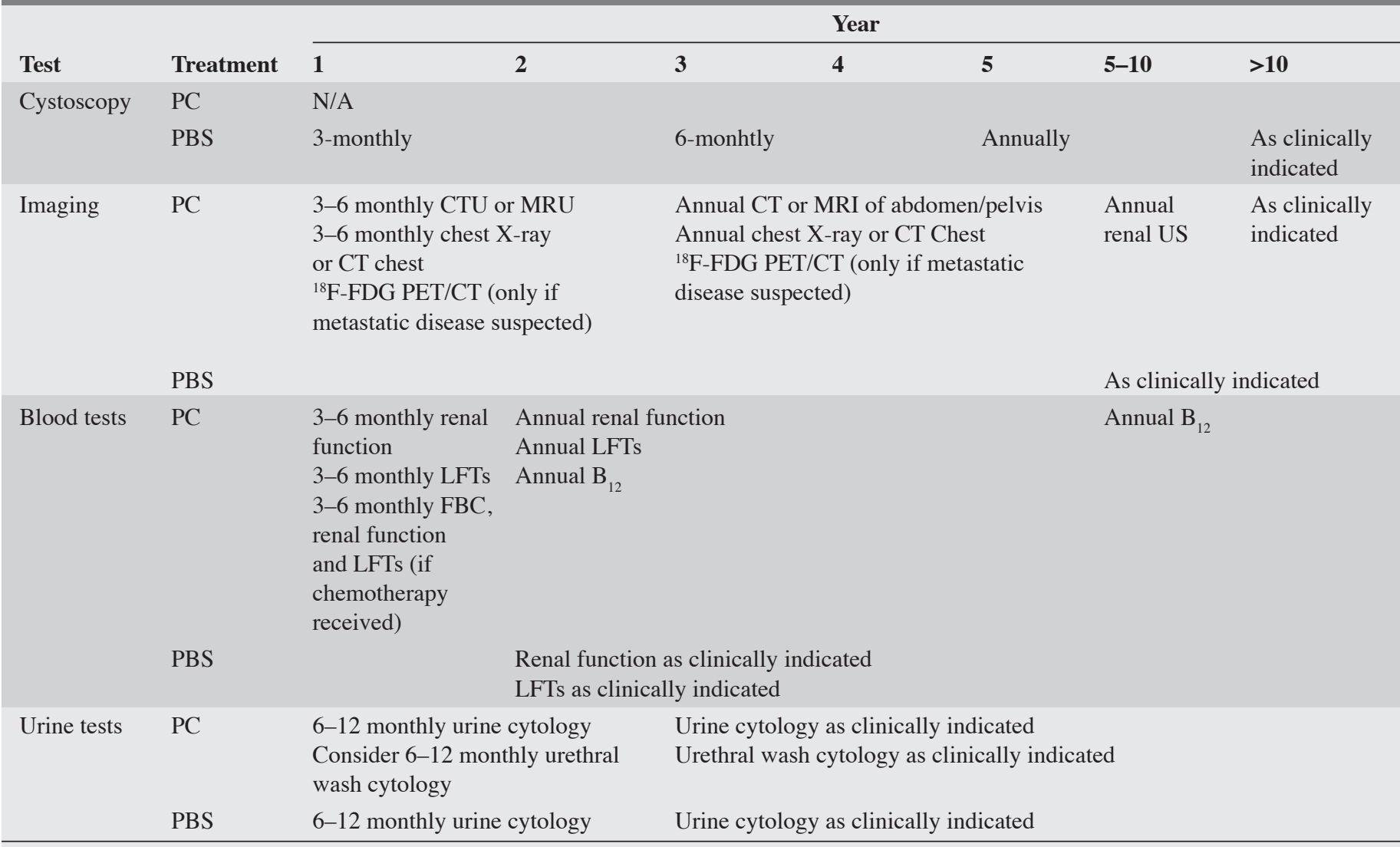

NCCN: National Comprehensive Cancer Network; PC: post-cystectomy; PBS: post-bladder sparing; CT: computed tomography; CTU: CT urography; MRU: magnetic resonance urography; ${ }^{18} \mathrm{~F}$-FDG-PET/CT: 18F-fluorodeoxyglucose-positron emission tomography/CT; LFTs: liver function tests; FBC: full blood count

\section{Adjuvant therapies}

In patients with MIBC who have not received neoadjuvant therapy, adjuvant cisplatin-based chemotherapy, chemoradiotherapy, or radiotherapy can be considered depending on which guideline is followed.

\section{Unresectable tumor management}

There is a consensus among the 4 major guidelines that in cases of locally advanced disease (T4b), no curative approach exists. Instead, patients should receive palliative treatments that are aimed at symptom relief. Options considered are palliative radiotherapy, chemotherapy, chemoradiotherapy, or, as a last resort, palliative cystectomy. However, the latter confers the greatest morbidity.

\section{Surveillance}

The surveillance plans suggested by the 4 major guidelines are summarized in Tables 2 and 3, with the NCCN guideline being the most comprehensive.

\section{Conclusion}

The majority of the recommendations show significant consensus among the guidelines because all of them are based on high- quality evidence and expert opinions. In areas of discrepancies, the clinicians should follow a holistic approach catering to the individual patient characteristics to adopt the most appropriate management strategy on a case-by-case basis.

Peer-review: Externally peer-reviewed.

Author Contributions: Concept - N.P.O., J.C.P.P., N.V.; Design N.P.O., J.C.P.P., N.V.; Supervision - N.V.; Resources - N.V.; Materials - N.P.O., J.C.P.P., N.V.; Data Collection and/or Processing - N.P.O., J.C.P.P., N.V.; Analysis and/or Interpretation - N.P.O., J.C.P.P., N.V.; Literature Search - N.P.O., J.C.P.P., N.V.; Writing Manuscript - N.P.O., J.C.P.P., N.V.; Critical Review - N.P.O., J.C.P.P., N.V.

Conflict of Interest: The authors have no conflicts of interest to declare.

Financial Disclosure: The authors declared that this study has received no financial support.

\section{References}

1. Chang SS, Bochner BH, Chou R, Dreicer R, Kamat AM, Lerner SP, et al. Treatment of Non-Metastatic Muscle-Invasive Bladder Cancer: AUA/ ASCO/ASTRO/SUO Guideline. J Urol 2017;198:552-9. [Crossref] 
2. Nice. National Collaborating Centre for Cancer Bladder cancer Bladder cancer: diagnosis and management NICE Guideline 2 Bladder cancer: diagnosis and management Contents. 2015.

3. Witjes JA, Bruins M, Cathomas R, Compérat E, Cowan NC, Gakis $\mathrm{G}$, et al. EAU Guidelines on Muscle-invasive and metastatic Bladder Cancer 2020 [Internet]. In: European Association of Urology Guidelines. 2020 Edition. European Association of Urology Guidelines Office; 2020 [cited 2020 May 21]. Available from: http://uroweb.org/guideline/bladder-cancer-muscle-invasive-andmetastatic/ LK - Muscle-invasive and Metastatic Bladder Cancer Uroweb\%7Chttp://uroweb.org/guideline/bladder-cancer-muscleinvasive-and-metastatic/\%7C FG - 0

4. Flaig TW, Spiess PE, Chair V, Agarwal N, Bangs R, Patient Advocate M, et al. NCCN Guidelines Version 5.2020 Bladder Cancer. 2020.

5. Eble JN. World Health Organization Classification of Tumours. Pathol Genet Tumours Urin Syst Male Genit Organs 2004;10.

6. Mostofi FK. International classification of tumours. Histol Classif Urin Bl tumours 1973;

7. Soria F, Krabbe LM, Todenhöfer T, Dobruch J, Mitra AP, Inman BA, et al. Molecular markers in bladder cancer. World J Urol 2019;37:31-40. [Crossref]

8. Wijkström H, Norming U, Lagerkvist M, Nilsson B, Näslund I, Wiklund P. Evaluation of clinical staging before cystectomy in transitional cell bladder carcinoma: A long-term follow-up of 276 consecutive patients. Br J Urol 1998;81:686-91. [Crossref]

9. Mariappan P, Zachou A, Grigor KM. Detrusor Muscle in the First, Apparently Complete Transurethral Resection of Bladder Tumour Specimen Is a Surrogate Marker of Resection Quality, Predicts Risk of Early Recurrence, and Is Dependent on Operator Experience. Eur Urol 2010;57:843-9. [Crossref]

10. Daneshmand S, Bazargani ST, Bivalacqua TJ, Holzbeierlein JM, Willard B, Taylor JM, et al. Blue light cystoscopy for the diagnosis of bladder cancer_ Results from the US prospective multicenter registry. Urol Oncol 2018;36:361 e1-361.e6. [Crossref]

11. Zang Z, Wu Q, Chiong E. Blue-light cystoscopy and narrowband imaging in bladder cancer management. Formos J Surg 2019;52:155. [Crossref]

12. Maruschke M, Kram W, Zimpfer A, Kundt G, Hakenberg OW. Upper Urinary Tract Tumors: Which Diagnostic Methods Are Needed? Urol Int 2017;98:304-11. [Crossref]

13. Schulz GB, Gresser EK, Casuscelli J, Strittmatter F, Tritschler S, Karl A, et al. Value of imaging in upper urinary tract tumors. Urol 2019;58:5-13. [Crossref]

14. Raitanen MP, Aine R, Rintala E, Kallio J, Rajala P, Juusela H, et al. Differences between local and review urinary cytology in diagnosis of bladder cancer. An interobserver multicenter analysis. Eur Urol 2002;41:284-9. [Crossref]

15. Van Rhijn BWG, Van Der Poel HG, Van Der Kwast TH. Urine markers for bladder cancer surveillance: A systematic review. Eur Urol 2005;47:736-48. [Crossref]

16. Barkan GA, Wojcik EM, Nayar R, Savic-Prince S, Quek ML, Kurtycz DFI, et al. The Paris System for Reporting Urinary Cytology: The Quest to Develop a Standardized Terminology. Acta Cytol 2016;60:185-97. [Crossref]
17. Brierley J, Gospodarowicz MD, Wittekind CT. TNM Classification of Malignant Tumors International Union Against Cancer. 8th. Oxford, England: Wiley; 2017. Oesphagus including oesophagogastric junction. Wiley 2017.pp.57-62. [Crossref]

18. Jensen JB, Høyer S, Jensen KME. Incidence of occult lymph-node metastasis missed by standard pathological examination in patients with bladder cancer undergoing radical cystectomy. Scand J Urol Nephrol 2011;45:419-24. [Crossref]

19. Capalbo E, Kluzer A, Peli M, Cosentino M, Berti E, Cariati M. Bladder cancer diagnosis: The role of CT urography. Tumori 2015;101:412-7. [Crossref]

20. Woo S, Suh CH, Kim SY, Cho JY, Kim SH. Diagnostic performance of MRI for prediction of muscle-invasiveness of bladder cancer: A systematic review and meta-analysis. Eur J Radiol 2017;95:46-55. [Crossref]

21. Huang L, Kong Q, Liu Z, Wang J, Kang Z, Zhu Y. The diagnostic value of MR imaging in differentiating $t$ staging of bladder cancer: A meta-analysis. Radiology 2018;286:502-11. [Crossref]

22. Gandhi N, Krishna S, Booth CM, Breau RH, Flood TA, Morgan $\mathrm{SC}$, et al. Diagnostic accuracy of magnetic resonance imaging for tumour staging of bladder cancer: systematic review and metaanalysis. BJU Int 2018;122:744-53. [Crossref]

23. Salmanoglu E, Halpern E, Trabulsi EJ, Kim S, Thakur ML. Aglance at imaging bladder cancer. Clin. Transl. Imaging 2018;6:257-69. [Crossref]

24. Recommendations for cross-sectional imaging in cancer management, Second edition Bladder cancer and other urothelial tumours Faculty of Clinical Radiology [Internet]. [cited 2020 May 23]. Available from: www.rcr.ac.uk

25. Ito Y, Kikuchi E, Tanaka N, Miyajima A, Mikami S, Jinzaki M, et al. Preoperative Hydronephrosis Grade Independently Predicts Worse Pathological Outcomes in Patients Undergoing Nephroureterectomy for Upper Tract Urothelial Carcinoma. J Urol 2011;185:1621-6. [Crossref]

26. Cowan NC, Turney BW, Taylor NJ, McCarthy CL, Crew JP. Multidetector computed tomography urography for diagnosing upper urinary tract urothelial tumour. BJU Int 2007;99:1363-70. [Crossref]

27. Guidance on gadolinium-based contrast agent administration to adult patients Contents [Internet]. [cited 2020 May 23]. Available from: www.rcr.ac.uk

28. Ebner L, Bütikofer Y, Ott D, Huber A, Landau J, Roos JE, et al. Lung nodule detection by microdose CT versus chest radiography (standard and dual-energy subtracted). Am J Roentgenol 2015;204:727-35. [Crossref]

29. Brendengen M, Winderen M, Fossa SD. Clinical significance of routine pre-cystectomy bone scans in patients with muscle-invasive bladder cancer. Br J Urol 1996;77:36-40. [Crossref]

30. Anderson TS, Regine WF, Kryscio R, Patchell RA. Neurologic complications of bladder carcinoma: A review of 359 cases. Cancer 2003;97:2267-72. [Crossref]

31. Lauenstein TC, Goehde SC, Herborn CU, Goyen M, Oberhoff C, Debatin JF, et al. Whole-body MR imaging: Evaluation of patients for metastases. Radiology 2004;233:139-48. [Crossref]

32. O'Sullivan GJ. Imaging of bone metastasis: An update. World J Radiol 2015;7:202. [Crossref] 
33. Heindel W, Gübitz R, Vieth V, Weckesser M, Schober O, Schäfers M. The diagnostic imaging of bone metastases. Dtsch Arztebl Int 2014;111:741-7. [Crossref]

34. Shinagare AB, Ramaiya NH, Jagannathan JP, Fennessy FM, Taplin ME, Van Den Abbeele AD. Metastatic pattern of bladder cancer: Correlation with the characteristics of the primary tumor. Am J Roentgenol 2011;196:117-22. [Crossref]

35. Girard A, Rouanne M, Taconet S, Radulescu C, Neuzillet Y, Girma A, et al. Integrated analysis of 18F-FDG PET/CT improves preoperative lymph node staging for patients with invasive bladder cancer. Eur Radiol 2019;29:4286-93. [Crossref]

36. Jeong IG, Hong S, You D, Hong JH, Ahn H, Kim CS. FDG PETCT for Lymph Node Staging of Bladder Cancer: A Prospective Study of Patients with Extended Pelvic Lymphadenectomy. Ann Surg Oncol 2015;22:3150-6. [Crossref]
37. Solsona E, Iborra I, Collado A, Rubio-Briones J, Casanova J, Calatrava A. Feasibility of Radical Transurethral Resection as Monotherapy for Selected Patients with Muscle Invasive Bladder Cancer. J Urol 2010;184:475-81. [Crossref]

38. Bolenz C, Ho R, Nuss GR, Ortiz N, Raj GV, Sagalowsky AI, et al. Management of elderly patients with urothelial carcinoma of the bladder: Guideline concordance and predictors of overall survival. BJU Int 2010;106:1324-9. [Crossref]

39. Yin M, Joshi M, Meijer RP, Glantz M, Holder S, Harvey HA, et al. Neoadjuvant Chemotherapy for Muscle-Invasive Bladder Cancer: A Systematic Review and Two-Step Meta-Analysis. Oncologist 2016;21:708-15. [Crossref]

40. Gore JL, Lai J, Setodji CM, Litwin MS, Saigal CS. Mortality increases when radical cystectomy is delayed more than 12 weeks: Results from a surveillance, epidemiology, and end results-medicare analysis. Cancer 2009;115:988-96. [Crossref] 